International Journal of Pure and Applied Mathematics

Volume 116 No. 1 2017, 45-48

ISSN: 1311-8080 (printed version); ISSN: 1314-3395 (on-line version)

url: http://www.ijpam.eu

doi: 10.12732/ijpam.v116i1.4

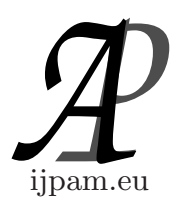

\title{
THE EULER FUNCTION GRAPH $G(\phi(n))$
}

\author{
S. Shanmugavelan \\ Department of Mathematics \\ Srinivasa Ramanujan Centre \\ SASTRA University Kumbakonam \\ 612001, INDIA
}

\begin{abstract}
The aim of this paper was to introduce the graph associated with the Euler's totient function and study its properties.
\end{abstract}

AMS Subject Classification: $05 \mathrm{C} 75,68 \mathrm{R} 10$

Key Words: Euler function graph, Euler function subgraph, Relatively prime graph, Euler totient cayley graph

\section{Introduction}

Euler totient function, also known as Euler's Phi function or simply Phi function, $\phi(n)$, for any natural number $\mathrm{n}$ in Number theory context represent the number of positive integer less than or equals $\mathrm{n}$ and relatively prime to $\mathrm{n}$. For example, $\phi(1)=1 ; \phi(7)=6$. Moreover, $\phi(n)$ is even for $n \geq 3$. Pomerance [6] defines the divisor graph to any non empty set $S$ of positive integers, where divisor graph $G(S)$ has vertex set $\mathrm{S}$ and any two vertices $i$ and $j$ are adjacent iff $\operatorname{gcd}(i, j)=\min (i, j)$. Certainly, $1 \leq \operatorname{gcd}(i, j)$. If equality of this considered, it makes him to define a relatively prime graph $R P(S)$ of $S$ having vertex set as $\mathrm{S}$ and any two vertices are adjacent iff they are co-primes. Further he showed that every graph is a relatively prime graph.

J. Baskar Babujee[1] investigated prime labelling for Euler's Phi function $\phi(n)$ and proved that a maximal number of edges in a simple vertex prime labeling graph with $\mathrm{n}$ vertices is $\sum_{k=2}^{n} \phi(n)$. Cayley graph associated with the

$\begin{array}{lr}\text { Received: } & \text { January 26, } 2017 \\ \text { Revised: } & \text { July 21, } 2017 \\ \text { Published: } & \text { August 29, } 2017\end{array}$

(c) 2017 Academic Publications, Ltd. url: www.acadpubl.eu 
Euler totient function, called Euler totient Cayley graph, whose vertex set V is given by $Z_{n}=\{0,1,2, \ldots, n-1\}$ and the edge set is $E=\{(x, y) / x-y \in S$ or $y-x \in S$ and is denoted by $\left(Z_{n}, \phi\right)$, where $S$ denote the set of all positive integers less than $\mathrm{n}$ and relatively prime to n. Moreover, Madhavi. L[3] proved that Euler totient function graph to be connected, Hamiltonian and Eulerian(for $n \geq 3$. M. Manjuri and B. Maheshwari[[4],[5]] proved the clique and matching domination number of $G\left(Z_{n}, \phi\right)$ for a prime is 1 and for prime powers to be 2 . K.J. Sangeetha, B. Maheswari[7] determined minimum edge cover, minimum edge dominating sets, edge covering number and edge domination number of $G\left(Z_{n}, \phi\right)$.

In this present paper, Euler function graph, a newer category of graph was introduced. Further, connectivity, bipartionness, completeness, Euler function subgraph was also discussed.

\section{Euler Function $G(\phi(n))$ Graph}

Now, Let us define a Euler function graph,

Definition 2.1. For any natural number $n$, Euler function graph $G(\phi(n))$ is a simple $(V, E)$ graph such that $V(G(\phi(n)))=\{a / g . c \cdot d(a, n)=1$ and $a<n\}$ and $E(G(\phi(n)))=\{a m / g . c . d(a, m)=1$ and $a<m$ or $m<a\}$.

Example 2.2. The Euler function graph for $n=1$ to 9 are

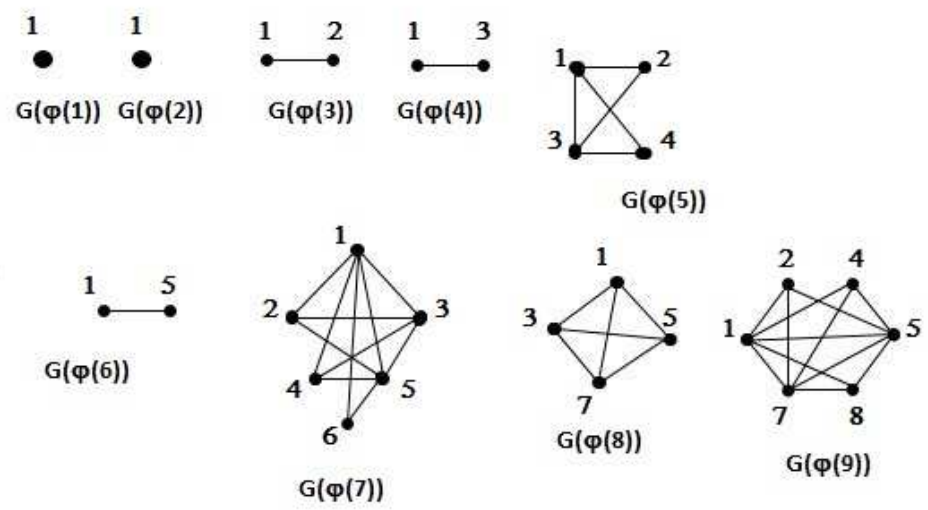

Remark 2.3. 1. The order of $G(\phi(n))$ is given by $n\left(1-\frac{1}{p_{1}}\right)\left(1-\frac{1}{p_{2}}\right) \ldots(1-$ $\left.\frac{1}{p_{k}}\right)$, where $\mathrm{n}$ can be expressed as a decomposition of positive integers as 


$$
n=p_{1}^{a_{1}} p_{2}^{a_{2}} \ldots p_{k}^{a_{k}}
$$

2. For any odd prime $p$, the order of $G(\phi(p))$ is exactly $(p-1)$ only and it is even.

3. Moreover, the maximum degree for $G(\phi(n)), \Delta=n\left(1-\frac{1}{p_{1}}\right)\left(1-\frac{1}{p_{2}}\right) \ldots(1-$ $\left.\frac{1}{p_{k}}\right)-1$ and if $p$ is odd prime, then the maximum degree is $p-2$.

4. Every Euler function graph is Relatively prime graph but the converse need not be true.

Theorem 2.4. For any positive integer $n$, the Euler function graph $G(\phi(n))$ is connected.

Proof. Clearly, $1 \in V(G(\phi(n))$ for any natural number $n$ and every integer is relatively prime to 1 . This means the vertex 1 is adjacent to all other remaining vertices in $G(\phi(n))$.

Corollary 2.5. Line graph of Euler function graph, $L(G(\phi(n)))$ is also connected.

Theorem 2.6. $G(\phi(n))$ is complete iff every pair of vertices of $G(\phi(n))$ are relatively prime.

Proof. Necessary part Assume that atleast one pair of vertices which are not relatively prime(say) $v_{1}, v_{2}$. Clearly, $v_{1}$ and $v_{2}$ are not adjacent in $G(\phi(n))$ and hence the resulting graph is not connected. Sufficient part It is obvious that if every pair of vertices of $G(\phi(n))$ are relatively prime, the graph is always connected.

Theorem 2.7. $G(\phi(n))$ is not Eulerian for any number $n$.

Proof. Clearly, for any $n \geq 3, \phi(n)$ is even and therefore there are even number of vertices in $G(\phi(n))$. Also, the vertex 1 is adjacent to all other vertices. This implies that $d_{G(\phi(n))}(1)=$ odd no. and hence the proof.

Definition 2.8. A subgraph $H$ of a Euler function graph $G(\phi(n))$ is called a Euler function subgraph if $H$ is itself a Euler function graph.

Example 2.9. Here $H_{1}, H_{2}$ are Euler function subgraph $G(\phi(3)), G(\phi(6))$ of a Euler function graph $G(\phi(5))$. 

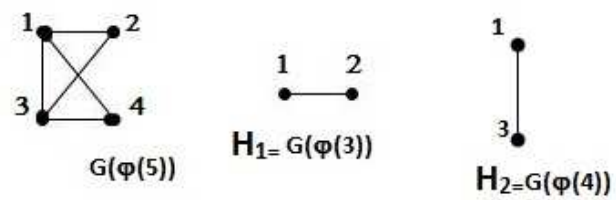

Remark 2.10. It is clear that every subgraph of $G(\phi(n))$ is neither a Euler function graph nor a relatively prime graph.

Theorem 2.11. If every pair of vertices in $G(\phi(n))$ are not relatively prime, then $G(\phi(n))$ is a bipartite graph.

Proof. Clearly, the vertex 1 is only element in any one of the partition (say)X and all other remaining vertices, which are not relatively prime to each other is in partition $\mathrm{Y}$. The resulting graph is a star $K_{1, p-1}$, where $p$ denotes the order of $G(\phi(n))$ and hence bipartite.

Remark 2.12. The following are some observations in $G(\phi(n))$

1. The Dominating number for $G(\phi(n))$ is 1 with minimal dominating set $\{1\}$.

2. The maximum no of edges in $G(\phi(p))$ cannot exceed $\left(\begin{array}{c}p-1 \\ 2\end{array}\right)$ or $\frac{(p-1)(p-2)}{2}$, for any prime $p$.

\section{References}

[1] J. Baskar Babujee, Euler's phi function and graph labeling, Int. J. Contemp. Math. Sciences, 5, No. 20, (2010), 977-984.

[2] K. Kannan, D. Narasimhan, S. Shanmugavelan, The graph of divisor function D(n), IJPAM, 102, No. 3 (2015), 483-494.

[3] L. Madhavi, Studies on Domination Parameters and Enumeration of Cycles in Some Arithmetic Graphs, Ph.D. Thesis, S.V. University, Tirupati, India, 2002.

[4] M. Manjuri, B. Maheswari, Clique dominating sets of euler totient cayley graphs, IOSR$J M, 4$, No. 6 (2013), 46-49.

[5] M. Manjuri, B. Maheswari, Matching dominating sets of Euler-Totient-Cayley graphs, IJCER, 2, No. 7 (2012), 103-107.

[6] C. Pomerance, On the longest simple path in the divisor graph, Cong. Numer. (1983), 291-304.

[7] K.J. Sangeetha, B. Maheswari, Edge domination in Euler-Totient-Cayley graph, IJSER, 3, No. 2 (2015), 14-17. 